\title{
miR-182-5p promotes hepatocellular carcinoma progression by repressing FOXO3a
}

Man-Qing Cao ${ }^{1,2}$, A-Bin You ${ }^{3}$, Xiao-Dong Zhu' ${ }^{1}$, Wei Zhang ${ }^{2}$, Yuan-Yuan Zhang ${ }^{1}$, Shi-Zhe Zhang ${ }^{1}$, Ke-wei Zhang ${ }^{4}$, Hao Cai ${ }^{1}$, Wen-Kai Shi ${ }^{1}$, Xiao-Long Li ${ }^{1}$, Kang-Shuai Li ${ }^{1}$, Dong-Mei Gao ${ }^{1}$, De-Ning Ma ${ }^{5}$, Bo-Gen Ye ${ }^{6}$, Cheng-Hao Wang ${ }^{7}$, Cheng-Dong Qin ${ }^{8}$, Hui-Chuan Sun ${ }^{1 *}$, Ti Zhang ${ }^{2^{*}}$ and Zhao-You Tang ${ }^{1 *}$

\begin{abstract}
Background: High frequency of recurrence is the major cause of the poor outcomes for patients with hepatocellular carcinoma (HCC). microRNA (miR)-182-5p emerged as a high-priority miRNA in HCC and was found to be related to HCC metastasis. Whether the expression of miR-182-5p in tumor tissue correlated with early recurrence in HCC patients underwent curative surgery was unknown.

Methods: Real-time PCR (RT-PCR) and in situ hybridization (ISH) were conducted to assess the expression of miR-182-5p in HCC cells and tissues. Cell Counting Kit-8 (CCK-8), transwell assays were performed to detected cells proliferation and migration ability. Flow cytometry assays were used to detect cell apoptosis rate, and xenograft model was employed to study miR-182-5p in HCC growth and lung metastasis. The target of miR-182-5p was validated with a dual-luciferase reporter assay and western blotting. Immunohistochemistry, immumoblotting, and immunoprecipitation were performed to test relative protein expression.

Results: We showed that high expression of miR-182-5p in tumor tissues correlated with poor prognosis as well as early recurrence in HCC patients underwent curative surgery. miR-182-5p enhanced motility and invasive ability of HCC cells both in vitro and in vivo. miR-182-5p directly targets 3'-UTR of FOXO3a and repressed FOXO3a expression, activating AKT/FOXO3a pathway to promote HCC proliferation. Notably, miR-182-5p activated Wnt/ $\beta$-catenin signaling by inhibiting the degradation of $\beta$-catenin and enhancing the interaction between $\beta$-catenin and TCF4 which was mediated by repressed FOXO3a.
\end{abstract}

Conclusions: Consistently, miR-182-5p can be a potential predictor of early recurrence for HCC patients underwent curative surgery, and FOXO3a plays a key mediator in miR-182-5p induced HCC progression.

Keywords: miR-182-5p, HCC, FOXO3a, Wnt signaling

\footnotetext{
* Correspondence: sun.huichuan@zs-hospital.sh.cn; zhangti@tjmuch.com; zytang88@163.com

'Department of Hepatobiliary Surgery, Liver Cancer Institute and Zhongshan Hospital, Fudan University, 180 Fenglin Road, Shanghai 200032, China ${ }^{2}$ Department of Hepatobiliary Surgery, Tianjin Medical University Cancer Institute and Hospital, National Clinical Research Center for Cancer, Tianjin's Clinical Research Center for Cancer, Key Laboratory of Cancer Prevention and Therapy, Tianjin 300060, China

Full list of author information is available at the end of the article
} 


\section{Background}

Hepatocellular carcinoma (HCC) is a prevalent malignancy that ranks the third leading cause of cancer mortality worldwide [1]. High frequencies of recurrence and metastasis are the major causes for the poor clinical outcomes of HCC patients. Increasing evidences have supported that miRNA deregulation is in correlation with $\mathrm{HCC}$ progression $[2,3]$. By targeting the $3{ }^{\prime}$ untranslated region (UTR) of the mRNA, miRNA leads to inhibition of targeted mRNA depending on total or partial complementarity $[4,5]$. miR-182-5p, a member of the miR-183/96/182 cluster, emerged as a highpriority miRNA in HCC and has been proven to be related to various cancers. However, the function of miR-182-5p is complicated because it can be an oncogene or a tumor suppressor in the context of different cancers. miR-182-5p is identified as onco-miR in ovarian cancer [6], breast cancer [7], and melanoma [8] and acts as tumor suppressor in $\operatorname{RCC}[9,10]$ and glioblastoma $[11,12]$. In HCC, miR-182-5p contributes to $\mathrm{HCC}$ metastasis by targeting metastasis suppressor 1 (MTSS1) [13], and upregulated miR-182$5 \mathrm{p}$ increases drug resistance in cisplatin-treated $\mathrm{HCC}$ cells by regulating tumor protein 53-induced nuclear protein 1 (TP53INP1) [14]. In addition, increased miR-182-5p can be of diagnostic and prognostic value in HCC patients [15]. However, whether miR-182-5p was involved in early recurrence of HCC remained unknown. We here investigated the relationship between miR-182-5p and early recurrence of HCC patients underwent curative surgery and further explored the underlying mechanisms of miR-182-5p in promoting $\mathrm{HCC}$ progression.

FOXO3a, a member of Forkhead box O (FOXO) transcription factor family, mediates many genes through its transcriptional activity, with important roles in cell fate decisions and is also suggested to play a pivotal role as a tumor suppressor in a wide range of cancers [16-18]. FOXO3a is an important target of PI3K/Akt pathway. Activated AKT phosphorylates FOXO3a and leads to its cytoplasmic translocation and subsequently degradation [19]. In addition to AKT, there are other negative regulators of FOXO3a, such as serum and glucocorticoidregulated kinase (SGK) [20, 21]. It has been reported that activation of the Wnt signaling pathway induced expression of SGK1 and lead to nuclear exclusion of FOXO3a [21], indicating that FOXO3a was under the regulation of $\mathrm{Wnt} / \beta$-catenin signaling pathway. In contrast, FOXO3a was reported to inhibit the expression $\beta$-catenin by transactivating $\mathrm{miR}-34 \mathrm{~b} / \mathrm{c}$ in prostate cancer [22], and FOXO3a can directly bind to $\beta$-catenin and compete with $\mathrm{T}$ cell factor (TCF) for the interaction to $\beta$ catenin, thereby inhibiting $\beta$-catenin/TCF transcriptional activity [23].
In the present study, we demonstrated that miR-182-5p could be a potential predictor for early recurrence of HCC patients underwent curative surgery, and miR-182-5p acted as a promoter of HCC growth both in vitro and in vivo. Notably, we found that miR-182-5p activated Wnt signaling pathway by inhibiting the degradation of $\beta$-catenin and enhancing the interaction between $\beta$-catenin and TCF4, which was mediated by repressed FOXO3a. These results provide new insight into the mechanism of miR-182-5p in promoting HCC progression.

\section{Methods}

\section{Cell culture and transfection}

HEK293T was originally obtained from the American Type Culture Collection (ATCC). MHCC-97H and MHCC-97L are human hepatocellular carcinoma cell lines with high metastatic potential [24], obtained from Liver Cancer Institute. All cell lines were maintained in Dulbecco's modified Eagle's medium supplemented with $10 \% \mathrm{FBS}, 10 \mathrm{U} / \mathrm{mL}$ penicillin, and $10 \mathrm{mg} / \mathrm{mL}$ streptomycin. Cells were grown in a humidified atmosphere at $37{ }^{\circ} \mathrm{C}$ at gas tensions of $20 \% \mathrm{O}_{2} / 5 \% \mathrm{CO}_{2}$ for normoxic incubation.

Plasmids used in the experiment:

miRNA inhibitor scrambled control: CmiR-AN0001AM03, anti-miR-182-5p: HmiR-AN0239-AM03, miR-1825p: HmiR0115-MR03, miR-NC: CmiR0001-MR03, and Lenti-Pac $^{\mathrm{Tm}}$ HIV Expression Packaging Kit (Cat.No. HPK-LvTR-20) were all purchased from the GeneCopoeia.

\section{Cellular proliferation assay and transwell assay}

For the cell proliferation assay, $5 \times 10^{3}$ cells were plated in 96-well plates. Cell growth was determined by using CCK8 assay. After transfection, MHCC-97H and MHCC-97L cells were suspended in $100 \mu \mathrm{L}$ serum-free medium and placed in the upper chambers of the transwell and incubated at $37{ }^{\circ} \mathrm{C}$ for $72 \mathrm{~h}$ for the invasion assay. The cells that penetrated the matrigel-coated filters were counted at a magnification of $\times 200$ in eight randomly selected fields, and the mean number of cells per field was recorded.

\section{Immunoprecipitation}

Whole-cell extracts were prepared in immunoprecipitation (IP) lysis buffer. The extracts were incubated overnight at $4{ }^{\circ} \mathrm{C}$ with $4 \mu \mathrm{g}$ antibody and for an additional $2 \mathrm{~h}$ with protein $\mathrm{A} / \mathrm{G}$ agarose beads. Beads were then washed three times with the lysis buffer, and the beads with the immunoprecipitates were resuspended in sodium dodecyl sulfate polyacrylamide gel electrophoresis (SDS-PAGE) sample buffer and heated at $95{ }^{\circ} \mathrm{C}$ for $10 \mathrm{~min}$. Subsequently, the supernatants were analyzed by SDS-PAGE followed by western blot. 


\section{Western blotting}

Western blot analyses were performed as previously described $[25,26]$.

AKT \#4685 CST; p-AKT Ser473 \#4060 CST; Bcl-2 \#15071; Bcl-xl \#2764. CST; Cyclin D1 \#2922 CST; SGK \#12103 CST; $\beta$-actin \#3700 CST; Wnt3a \#2721 CST; Wnt/ $\beta$-catenin Activated targets antibody Sampler Kit \#8655 CST; Forkhead Signaling Antibody Sampler Kit \#9946 CST.

\section{RNA preparation and real time-PCR (RT-PCR)}

Total RNA including miRNAs was extracted from cells using TRIzol reagent (Invitrogen) following the manufacturer's protocol. For analysis of miR-182-5p expression, reverse transcription and stem-loop RT-PCR were performed using the TaqMan MicroRNA assays (Applied Biosystems, Foster City, CA, USA) and amplified by TaqMan Universal PCR Master Mix (Applied Biosystems). U6 snRNA was probed as a loading control.

\section{In situ hybridization (ISH)}

ISH was used to detect miR-182-5p in tissue microarrays using digoxigenin-labeled sense and antisense miR-182-5p probes (Exiqon, 610341-360, Denmark). The slides were de-paraffined and rehydrated before incubation with Proteinase $\mathrm{K}$ at $37{ }^{\circ} \mathrm{C}$ for $40 \mathrm{~min}$, then wash the slides three times with PBS for 15 min. After incubation with $5 \times$ SSC solution at room temperature for $15 \mathrm{~min}$, miR-182$5 \mathrm{p}$ probes were added for hybridization at $50{ }^{\circ} \mathrm{C}$ for $1 \mathrm{~h}$. Next, the slides were washed with graded-diluted SSC solutions at $50{ }^{\circ} \mathrm{C}$ for $30 \mathrm{~min}$, followed by incubation with an antibody against digoxigenin (1:1000, Roche, Mannheim, Germany) at $4{ }^{\circ} \mathrm{C}$ overnight. Finally, hybridization signals were visualized by NBT/BCIP (Sigma), and the reaction was stopped by washing with water for $5 \mathrm{~min}$. At last, slides were counterstained with nuclear fast red for $1 \mathrm{~min}$ and then mounted using an aqueous solution.

\section{Dual-luciferase reporter assay}

The 3 '-UTR segments of FOXO3a including the wild type or the mutant type of miR-182-5p binding sites were cloned into the downstream of the luciferase reporter, the pmirGLO Dual-Luciferase miRNA Target Expression Vector (Promega, Madison, USA), between the SacI and Sall sites and verified by sequencing. HEK $293 \mathrm{~T}$ cells were plated into 24-well plates and transfected with $50 \mathrm{nM}$ miR-182-5p or NC and $100 \mathrm{ng}$ of the luciferase vector (pmirGLO). Cells were harvested $48 \mathrm{~h}$ after the transfection. The relative luciferase activity was measured by the Dual-Glo luciferase assay kit (Promega).

\section{Human samples}

This study was approved by the clinical research ethics committee of the Shanghai Zhongshan Hospital of Fudan
University. The tumor specimens for tissue microarrays were obtained from 119 patients who underwent surgery during July 2014 to May 2015 at the Shanghai Zhongshan Hospital of Fudan University. The disease-free survival was calculated from the date of resection to the date of tumor recurrence.

\section{Tumor xenografts in nude mice}

The orthotopic HCC implant tumor model in nude mice was performed as previously described [25, 26]. All surgical procedures and care administered to the animals were in accordance with the institutional ethics guidelines.

\section{Statistical analysis}

Immunohistochemistry (IHC) data were analyzed using a $\chi^{2}$ test. A two-tailed $t$ test was used to compare the means between two sets, and a one-way analysis of variance was used to compare the means among three groups. By the Kaplan-Meier method and the log-rank test, survival curve analysis was performed to study the role of miR-182-5p in HCC progression. The data were analyzed with SPSS software version 19.0 (SPSS Inc., Chicago, IL, USA). $P<0.05$ (two-sided) was considered statistically significant.

\section{Results}

miR-182-5p is upregulated in HCC cell lines and human HCC tissues, and its high expression predicts poor prognosis and early recurrence of $\mathrm{HCC}$

We firstly examined the expression level of miR-182-5p in several HCC cell lines and the normal liver cell line L02. miR-182-5p expressed relatively higher in HCC cell lines than in L02 cells (Fig. 1a). By using GEO database (GSE22058) [27], we compared the expression of miR182-5p in HCC and adjacent liver tissues. The mean level of miR-182-5p was higher in HCC tissues than that in adjacent liver tissues (Fig. 1b). We further analyzed the miR-182-5p expression in six paired HCC and adjacent tissues of patients in Liver Cancer Institute and Zhongshan Hospital, Fudan University; the mean level of miR-182-5p was also higher in HCC tissues than that in adjacent liver tissues (Fig. 1c). By employing the TCGA database, we further analyze the relationship between miR-182-5p and HCC prognosis. The results showed that patients with high miR-182-5p expression exhibited significantly shorter overall survival than patients with low miR-182-5p expression $(P<0.001)$ (Fig. 1d). ISH staining was used to detect the miR-182-5p expression in tissue microarray containing 119 human HCC patients with miR-182-5p probes (Fig. 1e). Patients with high miR-182-5p expression showed shorter recurrencefree survival than patients with low miR-182-5p expression (Fig. 1f). $(P=0.009$, Liver Cancer Institute and Zhongshan Hospital, Fudan University). 


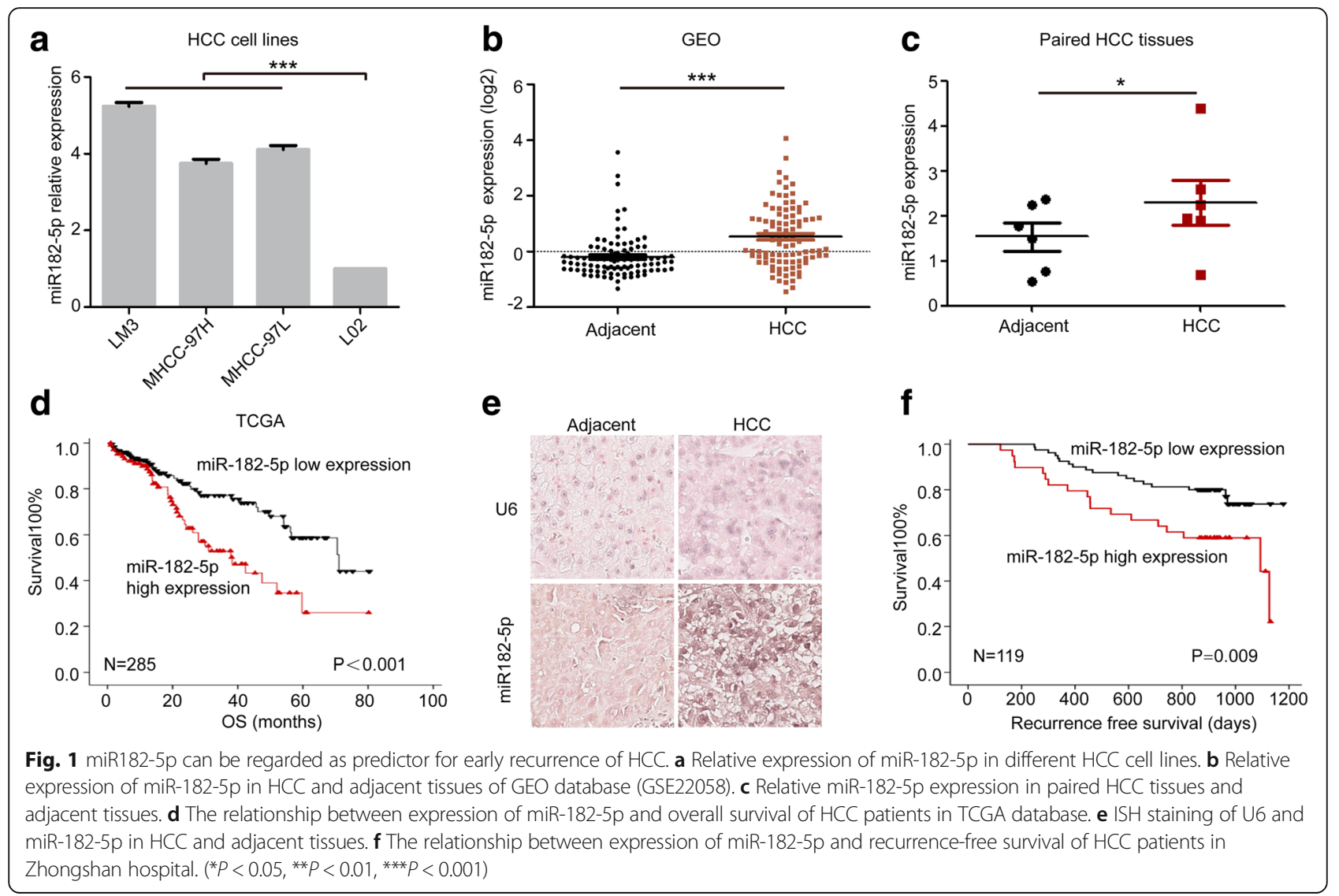

We further analyzed the correlation between miR-182-5p expression and clinicopathological parameters, and only AFP levels showed significant statistical difference (Table 1). Univariate analyses of prognostic factors for recurrencefree survivals showed that staining intensity of miR-182-5p, micro vein invasion (MVI), and AFP level had significant difference (Table 2). Multivariate analyses of prognostic factors for recurrence-free survivals showed that only the miR-182-5p staining intensity had the difference of statistics (Table 3). Taken together, these results implied that miR-182-5p could be a predictor for early recurrence of HCC.

miR-182-5p promotes HCC growth both in vitro and in vivo To investigate the effect of miR-182-5p on HCC, we transduced lentivirus to stably overexpress miR-182-5p expression in MHCC-97H cells and knockdown miR-182-5p in MHCC-97L cells (Fig. 2a). CCK8 assay showed that overexpression of miR-182-5p promoted HCC growth and clone formation ability in comparison with the control group. In contrast, knockdown miR-182-5p suppressed the growth of HCC cells (Fig. 2b). Based on the transwell assay, miR-182-5p overexpression significantly enhanced the invasion abilities of HCC cell, and knockdown miR-182-5p weakened the invasive ability of HCC cells (Fig. 2c). To further assess the function of miR-182-5p in tumor progression and metastasis, we used orthotropic transplantation model of HCC in nude mice. We evaluated the tumor sizes and lung metastases of these liver orthotropic models with different transfected cells. The tumor sizes and lung metastases of miR-182-5p overexpression group were greatly increased compared to the control group (Fig. 3a, c). The miR-182-5p knockdown group obviously delayed tumor sizes and number of lung metastases (Fig. 3b, d). Our data demonstrated that miR-182-5p played a role in the progression of HCC.

\section{miR-182-5p binds to 3 '-UTR of FOXO3a}

To identify specific gene targets of miR-182-5p through which it might promote oncogenic behavior in vitro and in vivo, the public algorithms (TargetScan, Pictar, miRANDA, miRWalk) were used. Among hundreds of predicted targets, FOXO3a was chosen not only for the reason that it was identified as an anti-oncogene but also because of its relatively good scores of predicted binding sites (Fig. 4a).

To verify the interaction between miR-182-5p and FOXO3a in HCC, luciferase reporter assay was performed. There are two binding site 72-79 site and 914-921 site of 3'-UTR of FOXO3a that could possibly bind with miR-182-5p (Fig. 4a). The wild-type vectors, two mutant 
Table 1 Correlation between miR-182-5p expression and HCC clinicopathological parameters

\begin{tabular}{|c|c|c|c|c|}
\hline \multirow[t]{2}{*}{ Parameters } & \multirow[t]{2}{*}{$n$} & \multicolumn{2}{|l|}{ miR-182-5p } & \multirow{2}{*}{$\begin{array}{l}P \\
\text { value }\end{array}$} \\
\hline & & Low $(n=80)$ & $\operatorname{High}(n=39)$ & \\
\hline \multicolumn{5}{|l|}{ Gender } \\
\hline Male & 100 & 66 & 34 & \multirow[t]{2}{*}{0.602} \\
\hline Female & 19 & 14 & 5 & \\
\hline \multicolumn{5}{|l|}{ Age (years) } \\
\hline$<50$ & 30 & 18 & 12 & \multirow[t]{2}{*}{0.372} \\
\hline$\geq 50$ & 89 & 62 & 27 & \\
\hline \multicolumn{5}{|l|}{ Cirrhosis } \\
\hline Absence & 27 & 22 & 5 & \multirow[t]{2}{*}{0.102} \\
\hline Presence & 92 & 58 & 34 & \\
\hline \multicolumn{5}{|c|}{ Tumor number } \\
\hline Single & 96 & 66 & 30 & \multirow[t]{2}{*}{0.471} \\
\hline Multiple & 23 & 14 & 9 & \\
\hline \multicolumn{5}{|c|}{ Tumor size(cm) } \\
\hline$<5 \mathrm{~cm}$ & 102 & 70 & 32 & \multirow[t]{2}{*}{0.418} \\
\hline$\geq 5$ & 17 & 10 & 7 & \\
\hline \multicolumn{5}{|c|}{ Micro vein invasion } \\
\hline Absence & 77 & 54 & 23 & \multirow[t]{2}{*}{0.416} \\
\hline Presence & 42 & 26 & 16 & \\
\hline \multicolumn{5}{|c|}{ HBV infection } \\
\hline Absence & 16 & 13 & 3 & \multirow[t]{2}{*}{0.259} \\
\hline Presence & 103 & 67 & 36 & \\
\hline \multicolumn{5}{|l|}{ AFP } \\
\hline$<20$ & 54 & 42 & 12 & \multirow[t]{2}{*}{$0.031^{*}$} \\
\hline$\geq 20$ & 65 & 38 & 27 & \\
\hline
\end{tabular}

AFP alpha-fetoprotein

${ }^{*} P<0.05$

3'-UTR of FOXO3a mRNA, and a double mutant vector were built to identify which sequence was responsible for binding to miR-182-5p. The luciferase activity of the wildtype vector group was significantly inhibited by miR-182$5 \mathrm{p}$. The luciferase activity in the FOXO3a mutant2 (M2) group was also inhibited, but luciferase activity of FOXO3a mutant1 (M1) group and M1 + M2 group was not suppressed by miR-182-5p mimics (Fig. 4a). These results indicated that miR-182-5p might inhibit FOXO3a expression by binding to $72-79$ site but not the 914-921 site of 3'-UTR of FOXO3a.

\section{miR-182-5p promotes HCC cells proliferation by activating AKT}

The underlying mechanisms of miR-182-5p on tumor growth were further explored. We observed a significant increase in AKT phosphorylation following miR-182-5p overexpression (Fig. 4b). In contrast, AKT phosphorylation
Table 2 Univariate analyses of prognostic factors for recurrencefree survivals

\begin{tabular}{|c|c|c|c|}
\hline Parameters & $n$ & Chi-square & $P$ value \\
\hline Gender & & 0.084 & 0.772 \\
\hline Male & 100 & & \\
\hline Female & 19 & & \\
\hline Age & & 0.009 & 0.924 \\
\hline$<50$ & 30 & & \\
\hline$\geq 50$ & 89 & & \\
\hline Cirrhosis & & 0.732 & 0.392 \\
\hline Absence & 27 & & \\
\hline Presence & 92 & & \\
\hline Staining intensity & & 6.728 & $0.009^{*}$ \\
\hline Low & 80 & & \\
\hline High & 39 & & \\
\hline Tumor number & & 1.177 & 0.278 \\
\hline Single & 96 & & \\
\hline Multiple & 23 & & \\
\hline Tumor size & & 0.000 & 0.986 \\
\hline$<5 \mathrm{~cm}$ & 102 & & \\
\hline$\geq 5$ & 17 & & \\
\hline Micro vein invasion & & 4.809 & $0.028^{*}$ \\
\hline Absence & 77 & & \\
\hline Presence & 42 & & \\
\hline HBV infection & & 0.861 & 0.353 \\
\hline Absence & 16 & & \\
\hline Presence & 103 & & \\
\hline AFP & & 5.859 & $0.015^{*}$ \\
\hline$<20$ & 54 & & \\
\hline$\geq 20$ & 65 & & \\
\hline
\end{tabular}

was inhibited by knockdown of miR-182-5p (Fig. 4b). Multiple studies have identified that FOXO3a was a downstream target of AKT [28]. To confirm AKT was activated, we further detected the expression of phosphorylation of FOXO3a in miR-182-5p overexpression and knockdown cells. Two sites of FOXO3a phosphorylation were both upregulated after miR-182-5p overexpression (Fig. 4b). Moreover, Bcl-2 and Bcl-xl, the downstream

Table 3 Multivariate analyses of prognostic factors for recurrence-free survivals

\begin{tabular}{lllll}
\hline Parameters & B & SE & $P$ value & Exp(B) \\
\hline Staining intensity & -0.713 & 0.341 & $0.036^{*}$ & 0.490 \\
Micro vein invasion & 0.554 & 0.345 & 0.108 & 1.740 \\
AFP & 0.592 & 0.382 & 0.121 & 1.808 \\
\hline
\end{tabular}

${ }^{*} P<0.05$ 

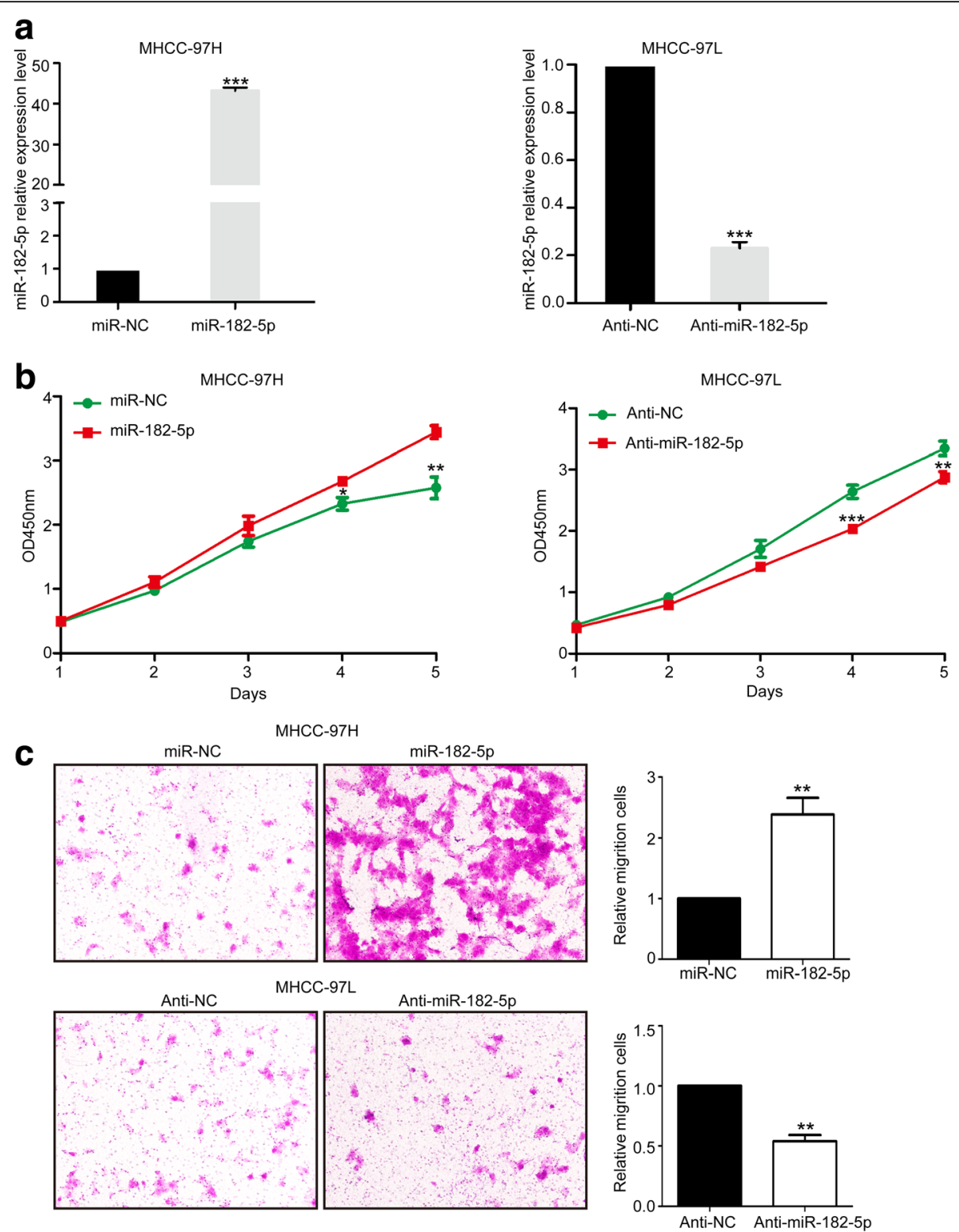

Fig. 2 Effects of miR182-5p in HCC proliferation and invasion. a Relative miR-182-5p expression in HCC cells that overexpressed miR-182-5p in MHCC-97H cells and knockdown miR-182-5p in MHCC-97L cells. b CCK8 assays of miR-NC cells and miR-182-5p-overexpression cells of MHCC-97H, and CCK8 assays of Anti-NC cells and miR-182-5p-inhibition cells of MHCC-97L cells. c Transwell assays of miR-NC cells and miR-182-5p-overexpression cells of MHCC-97H, and transwell assays of anti-NC cells and miR-182-5p-inhibition cells of MHCC-97L cells. $\left.{ }^{*} P<0.05,{ }^{* *} P<0.01,{ }^{* * *} P<0.001\right)$

target of AKT, were both upregulated following miR-182$5 \mathrm{p}$ overexpression (Fig. 4b). In order to explore FOXO3a was involved in increased phosphorylated AKT by miR-182-5p. We next established a stably FOXO3a knockdown cell line in MHCC-97H cells and measure the phosphorylation of AKT expression. The results showed that FOXO3a knockdown could greatly increase the expression of phosphorylation AKT, forming a positive feedback loop (Fig. 4c). As Bcl-2 and Bcl-xl were important anti-apoptosis proteins, we further detected the rate of apoptosis in miR-182-5p overexpression cells by fluorescence-activated cell sorting (FACS). Forced expression of miR-182-5p remarkably decreased the apoptosis rate (Fig. 4d). Thus, we proposed that overexpression of miR-182-5p activated AKT/FOXO3a signaling by repressing FOXO3a to promote proliferation of HCC cells.

\section{miR-182-5p promotes HCC metastasis by activating Wnt signaling}

Our results showed that forced miR-182-5p expression increased Wnt3a expression. Further, we found that the 

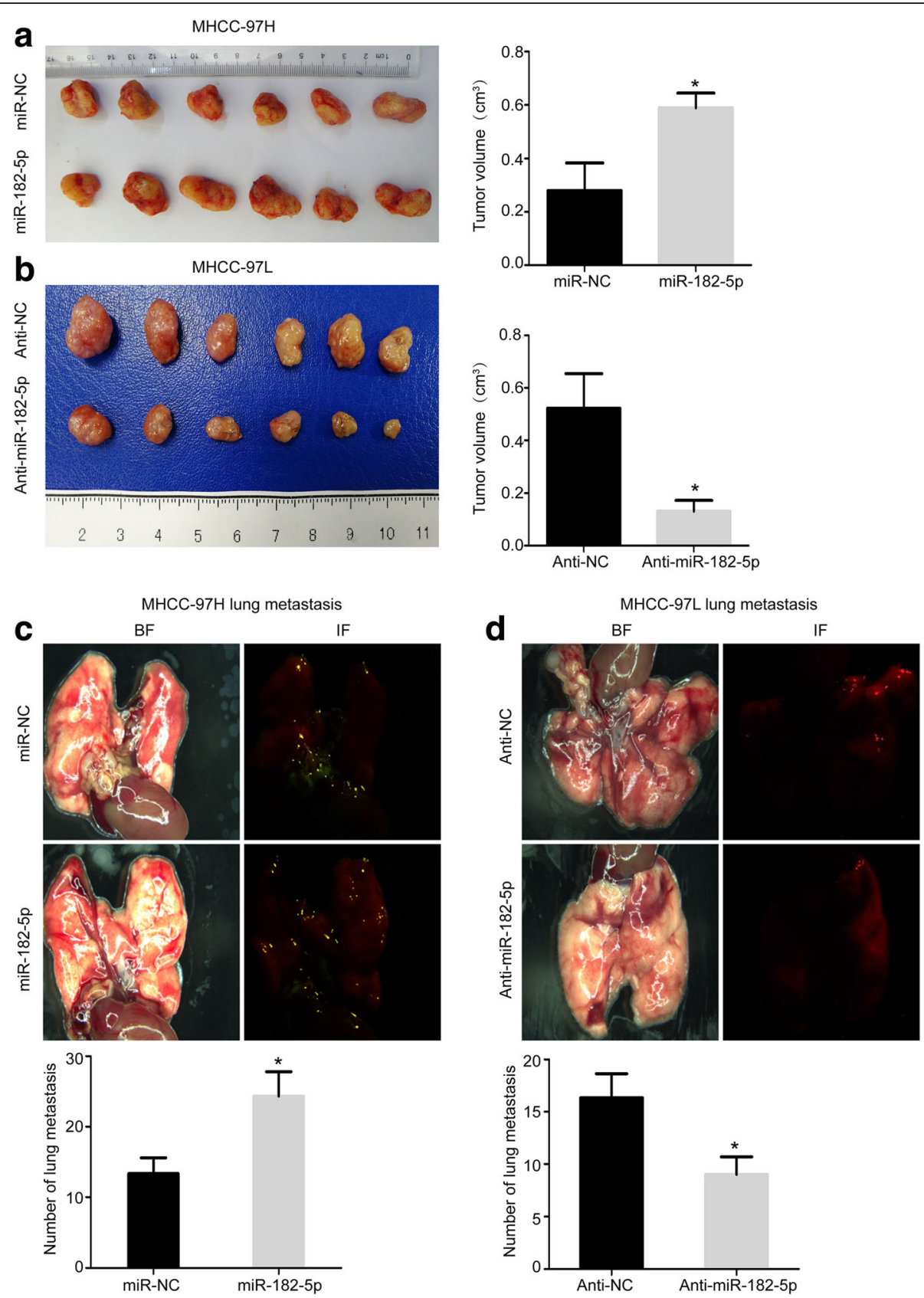

Fig. 3 The role of miR-182-5p in HCC growth and lung metastasis. a Orthotopic transplantation tumor formation with miR-NC and miR-182-5p overexpression in MHCC-97H cells, and tumor size analysis of the miR-NC and miR-182-5p overexpression groups. b Orthotopic transplantation tumor formation with anti-NC and miR-182-5p inhibition in MHCC-97L cells, and tumor size analysis of the anti-NC and miR-182-5p inhibition groups. c The images of lung metastasis of miR-NC and miR-182-5p-overexpression groups by stereo fluorescence microscope and lung metastasis analysis of miR-NC and miR-182-5p-overexpression groups. $\mathbf{d}$ The images of lung metastasis of anti-NC and miR-182-5p-inhibiton groups by stereo fluorescence microscope, and lung metastasis analysis of anti-NC and miR-182-5p-inhibition groups. $\left.{ }^{*} P<0.05,{ }^{* *} P<0.01,{ }^{* * *} P<0.001\right)$

downstream targets of Wnt signaling pathway were activated (Fig. 5a). By using IHC staining, we detected the expression of FOXO3a and $\beta$-catenin in orthotopic transplantation mice model tissues and showed that miR-182-5p remarkably suppressed FOXO3a whereas promoted $\beta$-catenin expression in HCC mouse model
(Fig. 5b). These results supported that Wnt signal pathway was activated by miR-182-5p.

In order to prove that Wnt signaling was involved in the enhanced metastasis ability by miR-182-5p, we used XAV939, a specific inhibitor of Wnt signaling pathway, to inhibit Wnt signal. Different concentration, 0,1,5,10,100, 
a
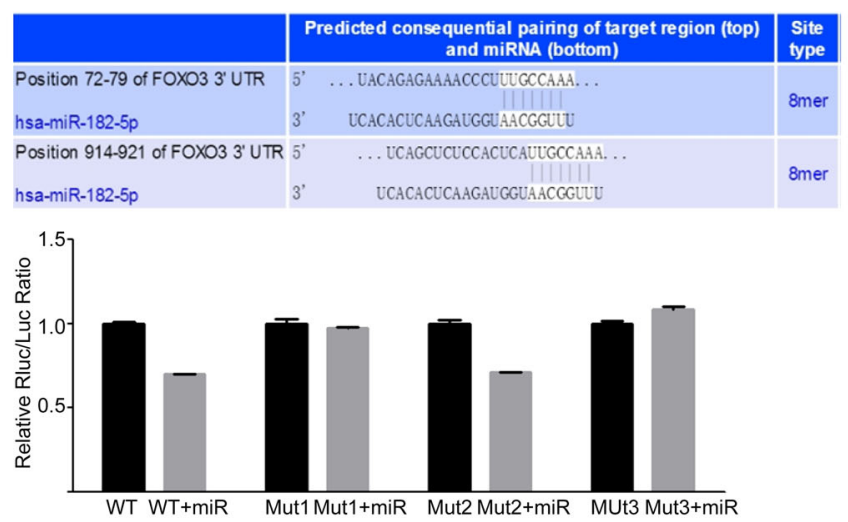

b
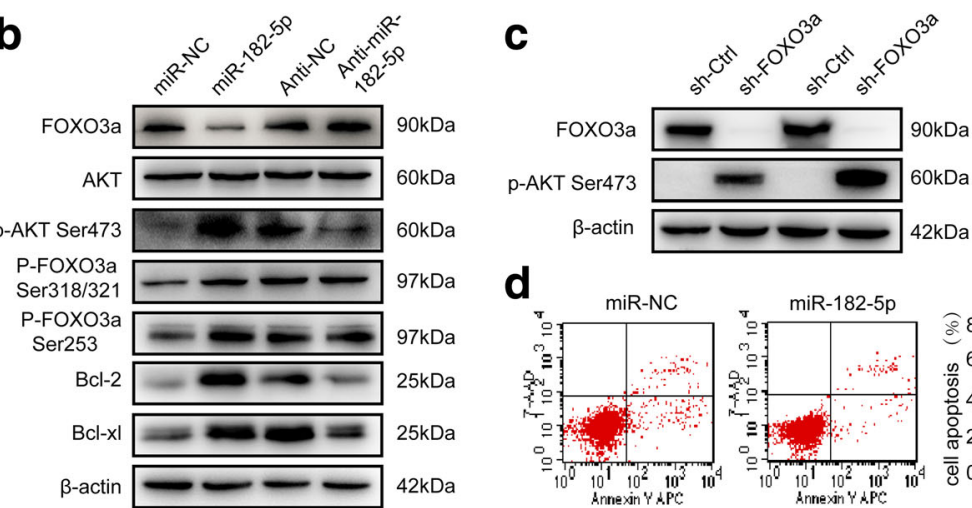

d

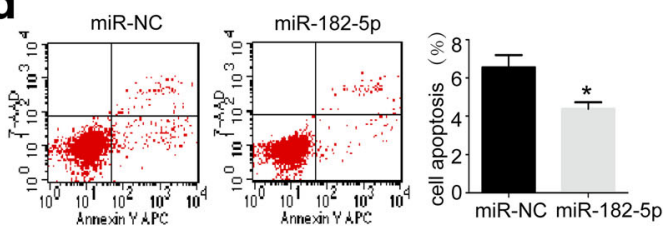

Fig. 4 miR-182-5p targets FOXO3a to activate AKT pathway. a Predicted binding sites of 3'-UTR of FOXO3a to miR-182-5p, and the relative Rluc/ Luc ratio of dual-luciferase reporter assay in different groups. $\mathbf{b}$ Western blot analysis of AKT/FOXO3a signaling-related proteins in control group, overexpression and knockdown miR-182-5p group of HCC cells. c Western blot analysis of p-AKT Ser473 expression after FOXO3a knockdown. $\mathbf{d}$ FASC analysis of cell apoptosis rate in miR-NC and miR-182-5p overexpression cells

and $200 \mu \mathrm{M}$, were used to treat HCC cells. After $12 \mathrm{~h}$, we detected the c-Myc expression, an important effector of Wnt signaling pathway and found that c-Myc gradually decreased along with the increasing concentration of XAV939 (Fig. 5c). We choose $5 \mu \mathrm{M}$ as a proper concentration for the subsequent treatment. Next, we employed the transwell assay to analyze the invasive ability of the control group, overexpression miR-182-5p group, and miR-182-5p overexpression with XAV939 group. The results showed that Wnt pathway inhibitor could restrain the invasiveness induced by miR-182-5p (Fig. 5d).

As FOXO3a has been proved to be related to Wnt signal regulation, we hypothesized that FOXO3a might play a role in the activation of Wnt signal pathway by miR-182-5p. We further overexpressed FOXO3a in miR-182-5p overexpression cells and detected the downstream targets of Wnt signaling pathway. The expression of Wnt3a and its downstream targets were suppressed compared to the miR-182-5p overexpressing cells (Fig. 5e), which implied that miR-182-5p activated the Wnt signaling pathway by modulating the expression of FOXO3a.
miR-182-5p activates Wnt signaling pathway through inhibiting $\beta$-catenin degradation and enhancing $\beta$-catenin/ TCF4 interaction via FOXO3a

$\beta$-catenin is a key inducer for the Wnt pathway, where in unstimulated cells, $\beta$-catenin proteins in the cytoplasm are phosphorylated and degraded by the proteasome $[29,30]$. Following Wnt signaling pathway activation, $\beta$-catenin was accumulated and localized into the nucleus to activate TCF/LEF transcription factors to initiate the expression of Wnt signaling target genes such as c-MYC and cyclin D1 [31]. To investigate how miR-182-5p activates Wnt signal pathway, we hypothesized that miR-182-5p overexpression might suppress $\beta$-catenin degradation. We therefore used protein synthesis inhibitor cycloheximide $(\mathrm{CHX})$ to treat the control cells and miR-182-5p overexpression cells. After 1.5 and $3 \mathrm{~h}$, the expression of $\beta$-catenin was measured by western blot. The degradation of $\beta$-catenin in the control cells was obviously faster than the miR-182-5p overexpression cells (Fig. 6a). Increasing evidences proved that FOXO3a can directly bind to $\beta$-catenin and competes with TCF for interaction with $\beta$-catenin $[22,23]$. By using the co-IP assays, we found that miR182-5p enhanced the interaction between 


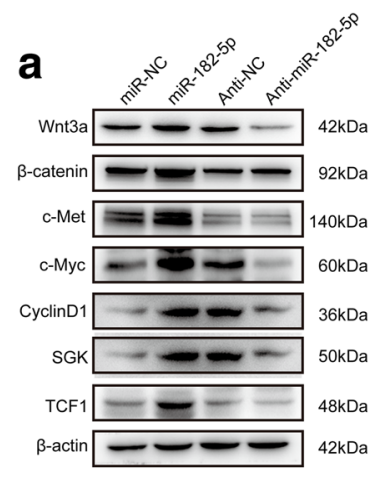

C
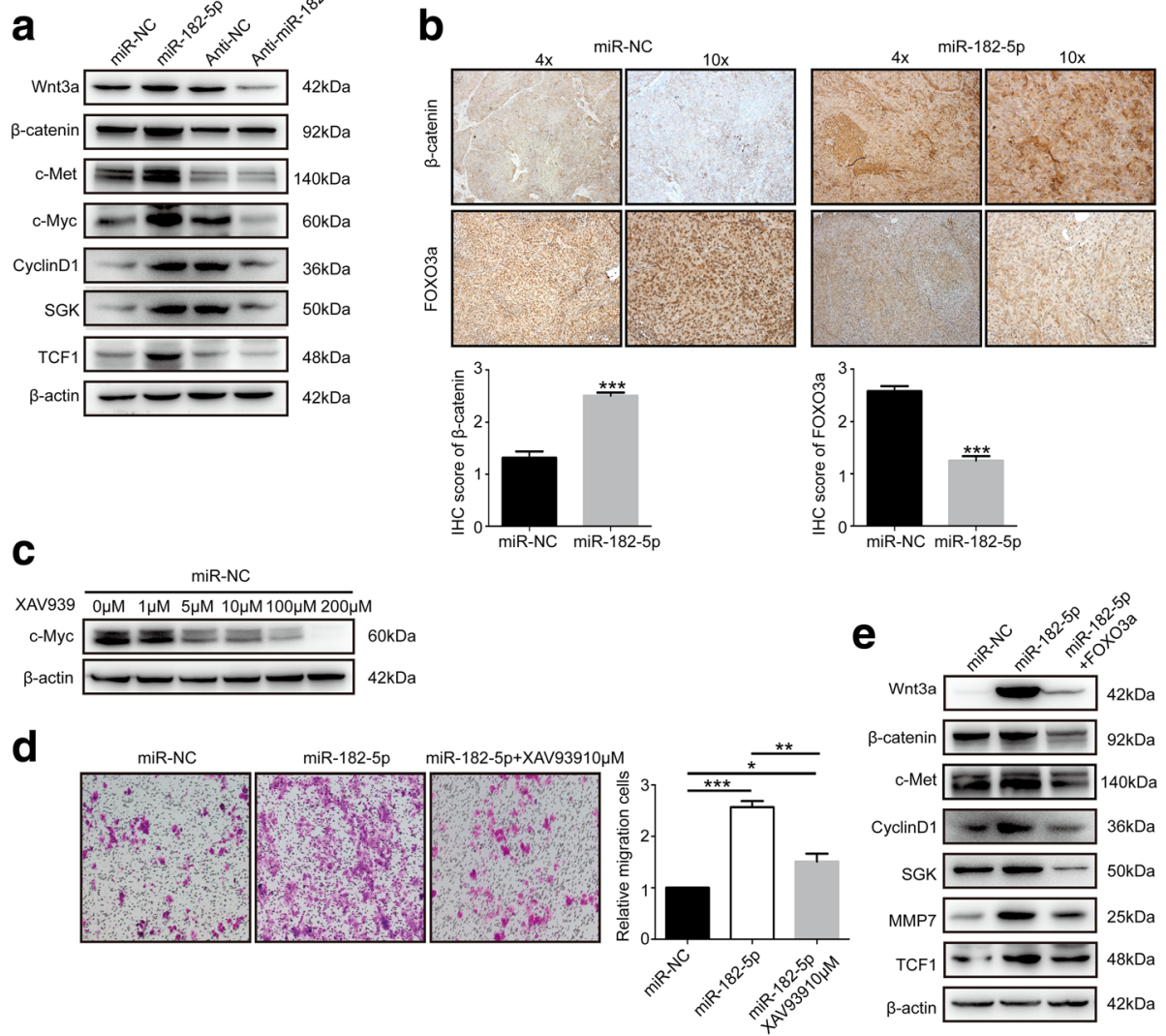

Fig. 5 FOXO3a exerts an important mediator in miR-182-5p induced Wht signaling activation. a Western blot analysis of Wnt signaling pathway related proteins following miR-182-5p overexpression and knockdown. $\mathbf{b} \mathrm{IHC}$ staining of FOXO3a and $\beta$-catenin in orthotopic tumor tissues of negative control and overexpression of miR-182-5p. c Western blot analysis of c-Myc in miR-NC cells treated with different concentration of XAV939 $(0,1,5,10,100$, and $200 \mu M)$. d Transwell assay of HCC cells that transfected with miR-NC, overexpression of miR-182-5p and miR-182-5p overexpression cells that further treated with $10 \mu \mathrm{M}$ XAV939. e Western blot analysis of Wnt signaling pathway-related proteins in miR-NC cells, overexpression of miR-182-5p cells and miR-182-5p overexpression cells further overexpress FOXO3a

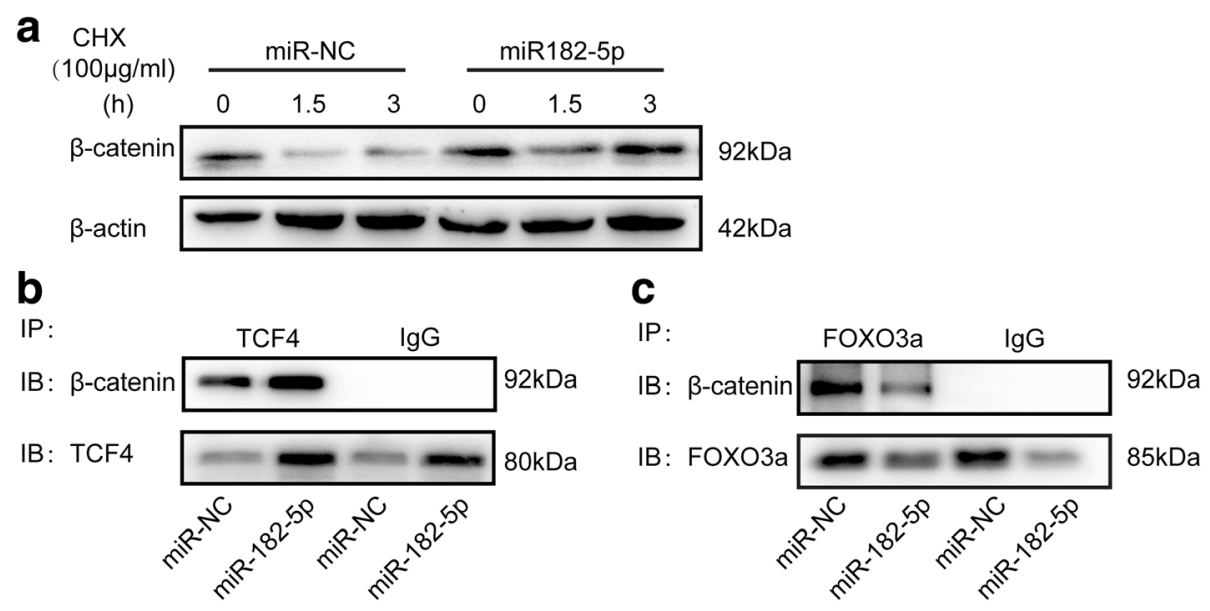

Fig. 6 miR-182-5p inhibits $\beta$-catenin degradation and enhances the binding of $\beta$-catenin/TCF4. a Western blot analysis of $\beta$-catenin expression in miR-NC and miR-182-5p overexpression cells that treated with $100 \mu \mathrm{g} / \mathrm{mL}$ CHX at 0, 1.5, and $3 \mathrm{~h}$. b Endogenous TCF4 was immunoprecipitated, and binding of $\beta$-catenin to TCF4 was analyzed by immunoblotting for $\beta$-catenin. $\mathbf{c}$ Endogenous FOXO3a was immunoprecipitated, and binding of $\beta$-catenin to FOXO3a was analyzed by immunoblotting for $\beta$-catenin 
$\beta$-catenin and TCF4, meanwhile, the interaction between $\beta$-catenin and FOXO3a was impaired (Fig. 6b). In conclusion, these results demonstrated that miR-182-5p activated Wnt signaling pathway by stabilization $\beta$-catenin and promoting $\beta$-catenin/TCF4 activity via inhibiting FOXO3a.

\section{Discussion}

Metastasis and recurrence are the main problems that limiting the 5 years' survival rate of HCC. In HCC patients, high-serum VEGF has been shown to associate with tumor recurrence, metastasis, and poor survival [32]. In addition to targeted drugs for VEGF in HCC, noninvasive detection of hydrodynamic changes in the field of industry has made a lot of progress [33, 34], which might be applied in the near future in the field of medicine.

Our results showed that miR-182-5p was overexpressed in both HCC cell lines and HCC tissues. Moreover, miR-182-5p was related to poor prognosis and early recurrence in HCC. By univariate and multivariate analyses, we found that the expression of miR-182-5p could be regarded a potential predictor for early recurrence of HCC patients under curative surgery.

miR-183, miR-182, and miR-96 act as regulators of FOXO expression in various cancer types [35, 36]. MiR-182-5p has been reported to promote melanoma metastasis by repressing FOXO3 [8]. However, the specific binding target site in the $3^{\prime}$-UTR was not showed. Here, we exhibited that miR-182-5p suppressed FOXO3a expression by binding to the 72-79 site, but not 914-921 site in the 3 '-UTR of FOXO3a.

Overexpression of miR-182-5p induces G1-phase arrest via inhibition of AKT/FOXO3a signaling in RCC [9]. However, we proved that miR-182-5p promoted AKT phosphorylation resulting in inactivation of FOXO3a in HCC. Moreover, knockdown of FOXO3a could further activate $\mathrm{AKT}$, indicating a positive feedback loop between $\mathrm{AKT}$ and FOXO3a. The apoptosis inhibiting protein $\mathrm{Bcl}-2$ and Bcl-xl, downstream target of AKT, were upregulated by overexpression of miR-182-5p. Taken together, miR-182-5p promoted cell proliferation by repressing FOXO3a, subsequently activating AKT/FOXO3a pathway.

It has been shown that Wnt/ $\beta$-catenin activated miR-183/96/182 expression in hepatocellular carcinoma and promoting cell invasion [37]. Furthermore, $\beta$-catenin enhances expression of primary and mature miR-96, miR-182, and miR-183 [38]. We proved that miR-182-5p activated Wnt signaling by inhibiting $\beta$-catenin degradation and promoting $\beta$-catenin/TCF4 interaction, forming a positive feedback loop between Wnt signaling and miR-182-5p.

Wnt/ $\beta$-catenin signaling pathway plays a critical role in the proliferation and cell cycle regulation of $\mathrm{HCC}$ cells [39]. When Wnt signaling is activated, $\beta$-catenin accumulates in the nucleus interacting with the $\mathrm{T}$ cell factor/lymphoid enhancer-binding factor (TCF/LEF) to transcriptionally activate downstream gene expression, such as cyclin D and c-Myc which exert promoting role in cell cycle $[31,40]$. $\beta$-catenin directly binds to FOXO and enhances FOXO transcriptional activity which is particularly important under condition of oxidative stress [41]. FOXO3a competes with TCF for interaction with $\beta$-catenin, thereby inhibiting TCF transcriptional activity [23]. Thus, $\beta$-catenin appears function as a switch that determining whether a cell chooses TCF or FOXO3a in response to stimuli.

We showed that miR-182-5p activated Wnt signaling by inhibiting $\beta$-catenin degradation, and miR-182-5p negatively regulate FOXO3a, resulting in the enhancement of $\beta$-catenin/TCF interaction, increasing the expression of $\beta$-catenin targeted genes. As a downstream factor of Wnt signaling, SGK1 preferentially phosphorylates Ser315, and AKT mediates the phosphorylation of Ser253 of FOXO3a [42]. SGK1 negatively regulates the transcription factor FOXO3a via phosphorylation and exclusion from the nucleus, leading to FOXO3a degradation [21]. When Wnt signal was activated by miR-182-5p, SGK was upregulated and lead to the phosphorylation of FOXO3a. As a result, the inactivation of FOXO3a might further promote Wnt signal, forming a positive feedback loop.

\section{Conclusions}

In conclusion, our results proved that miR-182-5p acted as a promoter in HCC and could be regarded as predictor for early recurrence of HCC patients underwent curative surgery. miR-182-5p negatively modulate FOXO3a by targeting 3'-UTR of mRNA in 72-79 site. We also proposed that FOXO3a played a key mediator in miR-182-5p induced malignant behaviors of $\mathrm{HCC}$, which may provide new insight into the mechanism of miR-182-5p in HCC progression.

\section{Abbreviations \\ ATCC: American Type Culture Collection; CCK-8: Cell Counting Kit-8; \\ FACS: Fluorescence-activated cell sorting; FOXO: Forkhead box O; \\ HCC: Hepotacellular carcinoma; IHC: Immunohistochemistry; \\ IP: Immunoprecipitation; LEF: Lymphoid enhancer-binding factor; \\ M1: Mutant1; M2: Mutant2; miR: MicroRNA; MTSS1: Metastasis suppressor 1; RT-PCR: Real time-PCR; SDS-PAGE: Sodium dodecyl sulfate polyacrylamide gel electrophoresis; SGK: Serum and glucocorticoid-regulated kinase; TCF: T cell factor; TP53INP1: Tumor protein 53-induced nuclear protein 1; \\ UTR: Untranslated region; VEGF: Vascular endothelial growth factor}

\section{Acknowledgements}

Not applicable.

\section{Funding}

This study was supported by the National Natural Science Foundation of China (No.81372635; No.81372655; No.81472224; No.81572434; No.81672326; and No.81672884) and National Science and Technology Major Project (No.2017ZX10203207).

Availability of data and materials

All data generated or analyzed during this study are included in this published article. 


\section{Authors' contributions}

MQC and ABY performed the experiments. WZ, YYZ, SZZ, KWZ HC, WKS, XLL, KSL, DMG, DNM, BGYe, CHW, and C-DQ analyzed the data. HCS, TZ, ZYT designed the research. MQC, XDZ, HCS, TZ, and ZYT wrote the manuscript. All authors read and approved the final manuscript.

\section{Ethics approval and consent to participate}

This study was approved by the clinical research ethics committee of the Shanghai Zhongshan Hospital of Fudan University.

\section{Consent for publication}

All the patients that involved in the study have given their consent to publish their individual data.

\section{Competing interests}

The authors declare that they have no competing interests.

\section{Publisher's Note}

Springer Nature remains neutral with regard to jurisdictional claims in published maps and institutional affiliations.

\section{Author details \\ 'Department of Hepatobiliary Surgery, Liver Cancer Institute and Zhongshan Hospital, Fudan University, 180 Fenglin Road, Shanghai 200032, China. ${ }^{2}$ Department of Hepatobiliary Surgery, Tianjin Medical University Cancer Institute and Hospital, National Clinical Research Center for Cancer, Tianjin's Clinical Research Center for Cancer, Key Laboratory of Cancer Prevention and Therapy, Tianjin 300060, China. ${ }^{3}$ Key laboratory of Carcinogenesis and Translational Research (Ministry of Education/Beijing), Division of Etiology, Peking University Cancer Hospital and Institute, Haidian District, Beijing 100142, China. ${ }^{4}$ Department of General Surgery, Xinhua Hospital, School of Medicine, Shanghai Jiao Tong University, Shanghai 200092, China. ${ }^{5}$ Department of Colorectal Cancer Surgery, Zhejiang Cancer Hospital, Zhejiang, Hangzhou 310022, China. ${ }^{6}$ Department of Organ Transplantation, Changhai Hospital, The Second Military Medical University, Shanghai 200433, China. ${ }^{7}$ Department of Liver Surgery, Fudan University Shanghai Cancer Center, Cancer Hospital, Shanghai 200032, China. ${ }^{8}$ Department of Breast Cancer Surgery, Zhejiang Cancer Hospital, Zhejiang, Hangzhou 310022, China.}

\section{Received: 15 December 2017 Accepted: 15 January 2018}

\section{Published online: 24 January 2018}

\section{References}

1. Torre LA, Bray F, Siegel RL, Ferlay J, Lortet-Tieulent J, Jemal A. Global cancer statistics, 2012. CA Cancer J Clin. 2015;65:87-108.

2. Budhu A, Jia HL, Forgues M, Liu CG, Goldstein D, Lam A, Zanetti KA, Ye OH, Qin LX, Croce CM, Tang ZY, Wang XW. Identification of metastasis-related microRNAs in hepatocellular carcinoma. Hepatology. 2008:47:897-907.

3. Ura S, Honda M, Yamashita T, Ueda T, Takatori H, Nishino R, Sunakozaka H, Sakai Y, Horimoto K, Kaneko S. Differential microRNA expression between hepatitis B and hepatitis C leading disease progression to hepatocellular carcinoma. Hepatology. 2009;49:1098-112.

4. Bartel DP. MicroRNAs: genomics, biogenesis, mechanism, and function. Cell. 2004;116:281-97

5. Krol J, Loedige I, Filipowicz W. The widespread regulation of microRNA biogenesis, function and decay. Nat Rev Genet. 2010;11:597-610.

6. Xu X, Ayub B, Liu Z, Serna VA, Oiang W, Liu Y, Hernando E, Zabludoff S, Kurita T, Kong B, Wei JJ. Anti-miR182 reduces ovarian cancer burden, invasion, and metastasis: an in vivo study in orthotopic xenografts of nude mice. Mol Cancer Ther. 2014;13:1729-39.

7. Li P, Sheng C, Huang L, Zhang H, Cheng Z, Zhu Q. MiR-183/-96/-182 cluster is up-regulated in most breast cancers and increases cell proliferation and migration. Breast Cancer Res. 2014;16:473.

8. Segura MF, Hanniford D, Menendez S, Reavie L, Zou X, Alvarez-Diaz S, Zakrzewski J, Blochin E, Rose A, Bogunovic D, Polsky D, Wei J, Lee P, Belitskaya-Levy I, Bhardwaj N, Osman I, Hernando E. Aberrant miR-182 expression promotes melanoma metastasis by repressing $\mathrm{FOXO} 3$ and microphthalmia-associated transcription factor. Proc Natl Acad Sci U S A. 2009;106:1814-9.
9. Xu X, Wu J, Li S, Hu Z, Zhu Y, Liang Z, Wang X, Lin Y, Mao Y, Chen H, Luo J, Liu B, Zheng $X$, Xie L. Downregulation of microRNA-182-5p contributes to renal cell carcinoma proliferation via activating the AKT/FOXO3a signaling pathway. Mol Cancer. 2014;13:109.

10. Wang X, Li H, Cui L, Feng J, Fan Q. MicroRNA-182 suppresses clear cell renal cell carcinoma migration and invasion by targeting IGF1R. Neoplasma. 2016; 63:717-25.

11. Kouri FM, Ritner C, Stegh AH. miRNA-182 and the regulation of the glioblastoma phenotype-toward miRNA-based precision therapeutics. Cell Cycle. 2015;14:3794-800

12. Kouri FM, Hurley LA, Daniel WL, Day ES, Hua Y, Hao L, Peng CY, Merkel TJ, Queisser MA, Ritner C, Zhang H, James CD, Sznajder Jl, Chin L, Giljohann DA, Kessler JA, Peter ME, Mirkin CA, Stegh AH. miR-182 integrates apoptosis, growth, and differentiation programs in glioblastoma. Genes Dev. 2015;29: 732-45.

13. Wang J, Li J, Shen J, Wang C, Yang L, Zhang X. MicroRNA-182 downregulates metastasis suppressor 1 and contributes to metastasis of hepatocellular carcinoma. BMC Cancer. 2012;12:227.

14. Qin J, Luo M, Qian H, Chen W. Upregulated miR-182 increases drug resistance in cisplatin-treated HCC cell by regulating TP53INP1. Gene. 2014; 538:342-7.

15. Chen L, Chu F, Cao Y, Shao J, Wang F. Serum miR-182 and miR-331-3p as diagnostic and prognostic markers in patients with hepatocellular carcinoma. Tumour Biol. 2015;36:7439-47.

16. Ni D, Ma X, Li HZ, Gao Y, Li XT, Zhang Y, Ai Q, Zhang P, Song EL, Huang QB, Fan $Y$, Zhang $X$. Downregulation of FOXO3a promotes tumor metastasis and is associated with metastasis-free survival of patients with clear cell renal cell carcinoma. Clin Cancer Res. 2014;20:1779-90.

17. Hu MC, Lee DF, Xia W, Golfman LS, Ou-Yang F, Yang JY, Zou Y, Bao S, Hanada N, Saso H, Kobayashi R, Hung MC. IkappaB kinase promotes tumorigenesis through inhibition of forkhead FOXO3a. Cell. 2004;117: 225-37.

18. Eijkelenboom A, Burgering BM. FOXOs: signalling integrators for homeostasis maintenance. Nat Rev Mol Cell Biol. 2013;14:83-97.

19. Guo S, Sonenshein GE. Forkhead box transcription factor FOXO3a regulates estrogen receptor alpha expression and is repressed by the Her-2/neu/ phosphatidylinositol 3-kinase/Akt signaling pathway. Mol Cell Biol. 2004;24: 8681-90.

20. Van Der Heide LP, Hoekman MF, Smidt MP. The ins and outs of FoxO shuttling: mechanisms of FoxO translocation and transcriptional regulation. Biochem J. 2004;380:297-309.

21. Dehner M, Hadjihannas M, Weiske J, Huber O, Behrens J. Wnt signaling inhibits Forkhead box O3a-induced transcription and apoptosis through up-regulation of serum- and glucocorticoid-inducible kinase 1. J Biol Chem. 2008:283:19201-10.

22. Liu $H$, Yin J, Wang $H$, Jiang G, Deng M, Zhang G, Bu X, Cai S, Du J, He Z. FOXO3a modulates WNT/beta-catenin signaling and suppresses epithelial-to-mesenchymal transition in prostate cancer cells. Cell Signal. 2015:27:510-8

23. Hoogeboom D, Essers MA, Polderman PE, Voets E, Smits LM, Burgering BM. Interaction of FOXO with beta-catenin inhibits beta-catenin/T cell factor activity. J Biol Chem. 2008;283:9224-30.

24. Li Y, Tang ZY, Ye SL, Liu YK, Chen J, Xue Q, Gao DM, Bao WH. Establishment of cell clones with different metastatic potential from the metastatic hepatocellular carcinoma cell line MHCC97. World J Gastroenterol. 2001;7: 630-6.

25. Cao M, Gao J, Zhou H, Huang J, You A, Guo Z, Fang F, Zhang W, Song T, Zhang T. HIF-2alpha regulates CDCP1 to promote PKCdelta-mediated migration in hepatocellular carcinoma. Tumour Biol. 2016;37:1651-62.

26. You A, Cao M, Guo Z, Zuo B, Gao J, Zhou H, Li H, Cui Y, Fang F, Zhang W, Song T, Li Q, Zhu X, Yin H, Sun H, Zhang T. Metformin sensitizes sorafenib to inhibit postoperative recurrence and metastasis of hepatocellular carcinoma in orthotopic mouse models. J Hematol Oncol. 2016;9:20.

27. Burchard J, Zhang C, Liu AM, Poon RT, Lee NP, Wong KF, Sham PC, Lam BY, Ferguson MD, Tokiwa G, Smith R, Leeson B, Beard R, Lamb JR, Lim L, Mao M, Dai H, Luk JM. microRNA-122 as a regulator of mitochondrial metabolic gene network in hepatocellular carcinoma. Mol Syst Biol. 2010;6:402.

28. Huang $\mathrm{H}$, Tindall DJ. Dynamic FoxO transcription factors. J Cell Sci. 2007;120: 2479-87.

29. Li VS, Ng SS, Boersema PJ, Low TY, Karthaus WR, Gerlach JP, Mohammed S, Heck AJ, Maurice MM, Mahmoudi T, Clevers H. Wnt signaling through 
inhibition of beta-catenin degradation in an intact Axin1 complex. Cell. 2012;149:1245-56.

30. Rao TP, Kuhl M. An updated overview on Wnt signaling pathways: a prelude for more. Circ Res. 2010;106:1798-806

31. Niehrs C, Acebron SP. Mitotic and mitogenic Wnt signalling. EMBO J. 2012; 31:2705-13.

32. Llovet JM, Pena CE, Lathia CD, Shan M, Meinhardt G, Bruix J, S.IS. Group. Plasma biomarkers as predictors of outcome in patients with advanced hepatocellular carcinoma. Clin Cancer Res. 2012;18:2290-300.

33. Li C.-x, J. Zhang, Nonlinear coupled dynamics analysis of a truss spar platform, China Ocean Engineering, 2016; doi:https://doi.org/10.1007/ s13344-016-0054-2.

34. Li C, Y. Liu, Fully-nonlinear simulation of the hydrodynamics of a floating body in surface waves by a high-order boundary element method, 2015; doi:https://doi.org/10.1115/OMAE2015-41448.

35. Myatt SS, Wang J, Monteiro L, Christian M, Ho KK, Fusi L, Dina RE, Brosens JJ, Ghaem-Maghami S, Lam EW. Definition of microRNAs that repress expression of the tumor suppressor gene FOXO1 in endometrial cancer. Cancer Res. 2010;70:367-77.

36. Guttilla IK, White BA. Coordinate regulation of FOXO1 by miR-27a, miR-96, and miR-182 in breast cancer cells. J Biol Chem. 2009;284:23204-16.

37. Leung WK, He M, Chan AW, Law PT, Wong N. Wnt/beta-catenin activates MiR-183/96/182 expression in hepatocellular carcinoma that promotes cell invasion. Cancer Lett. 2015:362:97-105.

38. Tang X, Zheng D, Hu P, Zeng Z, Li M, Tucker L, Monahan R, Resnick MB, Liu M, Ramratnam B. Glycogen synthase kinase 3 beta inhibits microRNA-18396-182 cluster via the beta-catenin/TCF/LEF-1 pathway in gastric cancer cells. Nucleic Acids Res. 2014;42:2988-98.

39. Qu C, He, Lu X, Dong L, Zhu Y, Zhao Q, Jiang X, Chang P, Wang L, Zhang Y, Bi L, He J, Peng Y, Su J, Zhang H, Huang H, Li Y, Zhou S, Qu Y, Zhao Y, Zhang Z. Salt-inducible kinase (SIK1) regulates HCC progression and WNT/ beta-catenin activation. J Hepatol. 2016;64:1076-89.

40. Reya T, Clevers H. Wnt signalling in stem cells and cancer. Nature. 2005;434: $843-50$

41. Essers MA, de Vries-Smits LM, Barker N, Polderman PE, Burgering BM, Korswagen HC. Functional interaction between beta-catenin and FOXO in oxidative stress signaling. Science. 2005;308:1181-4.

42. Brunet A, Park J, Tran H, Hu LS, Hemmings BA, Greenberg ME. Protein kinase SGK mediates survival signals by phosphorylating the forkhead transcription factor FKHRL1 (FOXO3a). Mol Cell Biol. 2001;21:952-65.

\section{Submit your next manuscript to BioMed Central and we will help you at every step:}

- We accept pre-submission inquiries

- Our selector tool helps you to find the most relevant journal

- We provide round the clock customer support

- Convenient online submission

- Thorough peer review

- Inclusion in PubMed and all major indexing services

- Maximum visibility for your research

Submit your manuscript at www.biomedcentral.com/submit

) Biomed Central 